\title{
Phenotyping Brachiaria Genotypes to Assess Rhizoctonia Resistance by Comparing Three Inoculum Types
}

Luis M. Hernandez, Guillermo Sotelo, Ximena Bonilla, Elizabeth Alvarez, John W. Miles, and Margaret Worthington, International Center for Tropical Agriculture (CIAT), Cali, Colombia

\begin{abstract}
Rhizoctonia foliar blight, caused by Rhizoctonia solani, is an important disease of Brachiaria spp. in tropical America. Host-plant resistance is an attractive option for disease management. In this study, we evaluated three inoculum types (mycelium-infected agar disc, microdiscs suspensions, and microencapsulatedmycelium suspensions) in order to identify a rapid and accurate method for mass screening of Brachiaria genotypes for resistance to Rhizoctonia spp. in greenhouse trials. Visual damage score, area under the disease progress curve, and percent chlorophyll loss were estimated to determine the most accurate and precise method for evaluating Rhizoctonia resistance. The microencapsulatedmycelium solution $(0.75 \mathrm{~g} / \mathrm{ml}$ in potato dextrose broth sprayed on plants

30 days after planting) caused greater foliar damage than the other inoculum types and allowed effective discrimination between resistant and susceptible genotypes. The effectiveness of spray-applied, microencapsulated-mycelium was further corroborated by the evaluation of 350 genotypes not previously selected for resistance to Rhizoctonia spp., which varied significantly in their reaction to $R$. solani. The microencapsulated-mycelium methodology has several advantages over existing methods, including its high-throughput capacity, efficient use of time and space, ease of quantification of inoculum, and consistent results over replicate trials. This methodology could be applied to assess resistance to Rhizoctonia spp. in other crops.
\end{abstract}

Forage grasses in the genus Brachiaria (Trin.) Griseb. (syn. Urochloa P. Beauv.) (Soreng et al. 2015) are widely used in sown pastures in the American tropics (Miles et al. 2004; Pabón et al. 2007; Peters et al. 2011). In Brazil alone, B. brizantha cv. Marandu occupies about 50 Mha (Jank et al. 2014). Different biotic [spittlebug complex (Hemiptera: Cercopidae) and Rhizoctonia solani Kühn] (Valerio et al. 1996; Miles et al. 2006; Cardona et al. 2010; Alvarez et al. 2013) and abiotic factors (drought, waterlogging and acid soils) (Rao et al. 2011) limit Brachiaria pasture productivity. Rhizoctonia foliar blight (RFB), caused by Rhizoctonia solani AG 1-1A, is considered the most important disease of Brachiaria grasses in Latin America (Valerio et al. 1996; Alvarez et al. 2014). Foliar damage caused by the fungus is reflected in biomass loss and consequently decreased feed availability. In the eastern plains of Colombia, RFB constitutes a plant health problem of economic importance (Peters et al. 2011; Alvarez et al. 2013).

For Rhizoctonia spp. management in other crops, different authors suggest cultural practices such as crop rotation and the removal of infected plant residues (Tsror 2010), but this is not practical for perennial pastures. Moreover, the capacity of the pathogen to survive for a long time as sclerotia or in infected plant debris makes disease management difficult (Alvarez et al. 2014). Therefore, implementation of preventive management strategies that minimize the losses caused by pathogens is necessary. In Brachiaria breeding for RFB resistance, different inoculum types have been employed (e.g., Sotelo et al. 2007, Kelemu et al. 1995). In more recent studies (e.g., Alvarez et al. 2013, 2014), plants have been inoculated with myceliuminfected agar discs, placed at junctions of leaf blades and leaf sheaths. Although this method is effective for the detection of resistant host genotypes, localized inoculation is impractical as it requires considerable time to inoculate large numbers of plants $(\sim 60$ plants/h per person), compared with spray microdiscs and microencapsulatedmycelium solutions ( $\sim 500$ plants/h per person).

Host-plant resistance is a practical, effective, environmentally sustainable, and economical method of plant disease control (Thakur 2007; Wallwork 2009). Research on plant disease resistance requires quick and cost-effective methodologies that allow discrimination

Corresponding Author: Elizabeth Alvarez; E-mail: e.alvarez@cgiar.org

Accepted for publication 18 January 2017.

() 2017 The American Phytopathological Society among genotypes differing in resistance; therefore, the development and optimization of suitable methodologies for the evaluation of resistance is essential in plant breeding programs. The properties of a desirable screening method are precise differentiation of genotypes and results that correlate with plant performance under relevant field conditions (Trapero et al. 2013). For this reason, screening methods to evaluate host-plant resistance to pathogens in the greenhouse must meet six criteria: (i) identification and use of the most virulent pathogen isolate(s) (Alvarez et al. 2013); (ii) use of an appropriate inoculum type, inoculation method, and suitable inoculation site (Park et al. 2008); (iii) determination and use of the optimal inoculum dose (Chongo and Bernier 2000); (iv) incubation under conditions appropriate for disease development (Park et al. 2008); (v) accurate assessment of disease development (Park et al. 2008); and (vi) consistency of results over replicate trials.

Various greenhouse methodologies have been developed to detect RFB resistance in crop breeding programs. To detect Rhizoctonia spp. resistance in rice (Oryza sativa L.), Araujo et al. (2007) found that inoculation of greenhouse-grown rice plants with infected rice grains scattered on the soil surface 64 days after sowing was a reliable method to quantify plant resistance. Maize (Zea mays L.) was also inoculated with rice grains infected with $R$. solani in the soil 15 days after planting (Pineda et al. 2005). However, disease progress using this method is slow, increasing evaluation time and the probability of disease escape. Thus, Park et al. (2008) determined that using liquidcultured mycelia balls as inoculum and covering the inoculated leaf sheaths with aluminum foil was the most reliable method to assess host-plant resistance to RFB in rice. Srinivasachary et al. (2011) suggested the hypothesis that two mechanisms contribute to Rhizoctonia spp. resistance in rice: Physiological resistance and disease escape. Willocquet et al. (2011) tested sclerotia as inoculum inserted below the leaf collar of individual rice tillers and maintained in tubes filled with water. This methodology allowed them to identify sources of resistance by the physiological mechanism. Srinivasachary et al. (2013) developed a biological model to identify sources of resistance combining microfield experiments and detached tiller assays.

Researchers (e.g., Jia et al. 2007; Sotelo et al. 2007; Park et al. 2008; Alvarez et al. 2014) have proposed inoculation of plants in microchambers (plastic bottles enclosing individual, inoculated plants with the aim of maintaining high relative humidity and physically isolating individual plants) with mycelium-infected agar discs as a suitable method for evaluating host plant resistance to RFB. However, in Brachiaria grasses, inoculation with mycelium-infected agar discs has three problems: (i) localized inoculation is impractical and excessive 
time is required to inoculate large numbers of plants; (ii) fungal colonization of the host plant is slow, and therefore more distal (upper) leaves may escape infection; and (iii) tall plants are not subject to the same inoculum pressure as short plants.

Therefore, the objective of this study was to standardize a robust, effective, repeatable, high-capacity, and low-cost methodology to discriminate variation in RFB reaction among Brachiaria genotypes under greenhouse conditions.

\section{Materials and Methods}

Experimental trials and plant material. This study was conducted in the Forage Pathology laboratories and greenhouses at the International Center for Tropical Agriculture (CIAT), in Palmira, Colombia $\left(3.5^{\circ} \mathrm{N} ; 76.4^{\circ} \mathrm{W}\right.$; elev. $1,000 \mathrm{~m} ; 24^{\circ} \mathrm{C}$ annual mean temperature). Two separate trials were conducted to: (i) assess the effectiveness of inoculation methods; (ii) validate the chosen inoculation methodology on a large breeding population. The experiment was conducted twice.

One susceptible genotype [Brachiaria hybrid 'Mulato', CIAT 36061] (Loch and Miles 2002) and one resistant genotype (B. brizantha, CIAT 16320) (Kelemu et al. 1995) were used in the first trial comparing inoculation methods. In the validation trial, additional checks [resistant (INRZ14/092), intermediate (INRZ14/061), and susceptible (INRZ05/3394) genotypes from recurrent selection population under selection for flooding tolerance and RFB resistance] and 350 clones of unknown reaction to RFB from the tenth cycle of a synthetic, tetraploid, sexually reproducing, allogamous Brachiaria recurrent selection population (SX14) were tested for RFB disease reaction. This population was originally synthesized from selected accessions representing three Brachiaria species: B. ruziziensis (high nutritional quality); $B$. brizantha (resistance to spittlebugs); and B. decumbens (resistance to low $\mathrm{pH}$ soils). Details of the origin of this recurrent selection population are described in Miles (2007). This population has not been subjected to intentional selection for RFB resistance.

Test plants in both trials were established by vegetative propagation from pot-grown mother plants maintained in an unheated greenhouse $\left(28^{\circ} \mathrm{C} ; 80 \% \mathrm{RH}\right)$. To minimize microbial contamination during vegetative propagation, propagules were immersed for $5 \mathrm{~min}$ in a $1 \%$ sodium hypochlorite solution. Each propagule was planted individually in a PVC unit (5.3 cm wide by $6.5 \mathrm{~cm}$ deep) containing $40 \mathrm{~g}$ of sterilized soil (3:1 soil:sand). These compact growing containers occupied little space $\left(125\right.$ plants $\left./ \mathrm{m}^{2}\right)$. These plant growth units were described and illustrated by Cardona et al. (1999). Plants were fertilized with $30 \mathrm{ml}$ of nutrient solution prepared with a $15-15-15$ soluble fertilizer at $3 \mathrm{~g} /$ liter two weeks after propagation and subsequently watered as needed. The experiments were conducted in the greenhouse and plants were inoculated beginning 30 days after propagation. At the time of inoculation the plants had one tiller and 3 to 4 leaves per tiller.

The fungal isolate A-36061 (Anastomosis group 1 and intraspecific group IA; $R$. solani AG 1-IA) (Alvarez et al. 2013) was used in all assays. This isolate, collected in 2012 from naturally inoculated plants of cv. Mulato growing in the field at the Macagual research station in the municipality of Florencia, Caquetá, Colombia (Alvarez et al. 2014), was previously described as the most virulent Colombian isolate of $R$. solani tested on Brachiaria spp. (Alvarez et al. 2013).

Inoculation methods trial. Inoculum preparation. Three inoculum types-mycelium-infected agar discs (Inoculum I) (Alvarez et al. 2014), microdiscs suspensions (Inoculum II), and microencapsulatedmycelium suspensions (Inoculum III) — were prepared in order to identify the best method of inoculation for evaluation of RFB reaction in Brachiaria isolates. The fungus, maintained as vegetative mycelia on filter paper at $-20^{\circ} \mathrm{C}$, was reactivated in Petri dishes $(15-\mathrm{cm}$-diameter with $60 \mathrm{ml}$ of potato dextrose agar medium (PDA) and incubated in the dark at $23^{\circ} \mathrm{C}$ for $72 \mathrm{~h}$ ). For inoculum I, 0.5-cm-diameter agar disc with mycelium were extracted excluding the central part of Petri dish, which contains older mycelia and sclerotia. Two concentrations ( 1 or 2 discs per plant) of this inoculum type were evaluated. For Inoculum II, the totality of Rhizoctonia-infected agar was removed from the Petri dish after growth for $72 \mathrm{~h}$ at $23^{\circ} \mathrm{C}$ and passed through a $0.1-\mathrm{cm}$ sieve to generate agar "microdiscs". The agar microdiscs were then dissolved in potato dextrose broth (PDB). Plants were then exposed to four concentrations of inoculant: $0.25 ; 0.50 ; 0.75$; or $1 \mathrm{~g} / \mathrm{ml}$ of agar microdiscs suspended in PDB.

For Inoculum III, Erlenmeyer flasks $(250 \mathrm{ml})$ with $200 \mathrm{ml}$ of PDB were inoculated with five 0.5 -cm-diameter mycelium-infected agar discs. After incubation for 7 days at $23^{\circ} \mathrm{C}$ in a shaker at $100 \mathrm{rpm}$, mycelium was triturated using a blender $(11,000 \mathrm{rpm}$ for $1 \mathrm{~min}$; Waring blender 7011 model 31BL92, Conair Corp., East Windsor, NJ). Mycelium was then separated from the liquid substrate by vacuum filtration using a Büchner funnel (250 ml, Beijing Getty Laboratory Glassware Co., Ltd) with Miracloth membrane (Calbiochem, San Diego, CA) and washed with distilled water using an aspirator. One hundred ml PDA was mixed with $2.3 \mathrm{~g}$ mycelium to produce a homogenous mixture of mycelium and agar and poured into Petri dishes. Following agar polymerization, the mycelium and agar mixture was removed from the Petri dish and passed through a $0.1-\mathrm{cm}$ sieve to generate "agar microencapsulated mycelium." Plants were then exposed to four concentrations of agar microencapsulated mycelium: 0.25 ; $0.50 ; 0.75$; or $1 \mathrm{~g} / \mathrm{ml} \mathrm{PDB.}$

Plant Inoculation. The three inoculum types were evaluated on two host genotypes: CIAT 36061, a known susceptible check, and CIAT 16320, previously determined to be RFB-resistant (Kelemu et al. 1995). The Inoculum I discs of mycelium on agar were carefully placed at the point of union between the leaf blade and the leaf sheath following Alvarez et al. (2014), starting from the most basal (oldest) leaf. Controls were inoculated with sterile agar disc(s). The agar solutions (Inoculum II and III) were sprayed uniformly on the plants (50 ml solution applied to 20 plants in a flat; Fig. 1A) using a manual pump (1.5-liter, Roots Garden Ergo, Shixia Holding Co, China). The controls consisted of untreated plants inoculated with a sterile agar microdiscs solution $(0.25 \mathrm{~g} / \mathrm{ml}$ PDB$)$, which were also evaluated for disease symptoms in order to determine the extent to which observed plant damage could be attributed to RFB. The absolute control consisted of noninoculated plants in microchambers. Inoculated plants were immediately introduced into individual microchambers (Fig. 1A, B, and C). The microchambers prevented physical contact between plants and maintained a high relative humidity (>90\%, unpublished data) and intensity of light of $50 \mu \mathrm{mol} / \mathrm{m}^{2} / \mathrm{s}$. Temperature and relative humidity inside microchambers were monitored continuously using a data logger (HOBO Pro V2, Onset Computers, Bourne, MA). The 20 treatment combinations (genotype, inoculation type, and dosage) were randomly assigned to 20 single-plant experimental units in each of 10 blocks in a randomized complete block (RCBD) design. In order to confirm the results of the first experiment and compare the optimum concentrations of the three inoculum types, the experiment was subsequently repeated with eight more replicates in a RCBD. The same genotypes (CIAT 36061 and CIAT 16320) were inoculated with agar discs (two agar discs), microdiscs $(0.75 \mathrm{~g} / \mathrm{ml} \mathrm{PDB})$, and agar microencapsulated mycelium $(0.75 \mathrm{~g} / \mathrm{ml}$ PDB) as previously described.

Disease evaluation. Plants were evaluated at three, five, and seven days after inoculation using an 11-point scale of visual damage, Visual Damage Score (VDS), as follows: $0=$ disease symptoms absent and $5.0=100 \%$ infected area. This is a scale for which grades 0.5 to 5 correspond to median severities of 5 to $95 \%$, with a $10 \%$ increase between each grade. Visual damage scores were used to calculate the area under the disease progress curve according to Shaner and Finney (1977):

$$
A U D P C=\sum_{i=1}^{n}\left[\frac{X_{i}+X_{i-1}}{2}\right]\left[t_{i}-t_{i-1}\right],
$$

where $X_{i}=$ visual damage score of disease at the $i$ th evaluation $\left(X_{O}=\right.$ $0), t_{i}=$ time in days from inoculation to the $i$ th evaluation $\left(\mathrm{t}_{0}=0\right)$, and $\mathrm{n}=$ number of evaluations. Leaf chlorophyll content in SPAD units was estimated one day before inoculation and eight days after inoculation with a SPAD 502 chlorophyll meter (Konica Minolta Sensing, Osaka, Japan). The SPAD values were converted to total chlorophyll content $(\mathrm{mg} / \mathrm{g})$ using the formula developed by Jiménez et al. (2015) for Brachiaria: ChlC (mg/g) $=0.4036$ (SPAD meter reading) -0.5527 . The percentage chlorophyll loss $(\mathrm{ChlL})$ was calculated 
as $[(\mathrm{ChlC} 1-\mathrm{ChlC} 2) / \mathrm{ChlC} 1] \times 100$, where $\mathrm{ChlC} 1=$ the average chlorophyll content one day before inoculation and $\mathrm{ChlC} 2=$ the average of chlorophyll content eight days after inoculation. The average chlorophyll content per plant was estimated by taking readings on three leaves per plant. The utility of VDS as an indicator of plant damage was validated by calculating Pearson's correlation coefficients between VDS scores (7 days after inoculation) and assessments of AUDPC and ChlL. The following response variables were subjected to analysis of variance (ANOVA) using SAS 9.2 (SAS Institute Inc., Cary, NC): VDS at 3, 5, and 7 days after inoculation, AUDPC, and ChlL. Separation of treatment means was performed using Fisher's protected least significant difference (LSD, $P<0.05$ ). Uninoculated control plants were not included in analyses.

Methodology. Three hundred and fifty genotypes from the SX14 population were used to validate the efficacy of inoculation with microencapsulated-mycelium suspension. Inoculum at a concentration of $0.75 \mathrm{~g} / \mathrm{ml}$ was prepared as previously described, with $26 \mathrm{~g}$ of mycelium encapsulated in $1131 \mathrm{ml}$ of potato dextrose agar, diluted in $1509 \mathrm{ml}$ of $\mathrm{PDB}$, and sprayed on 1065 plants 30 days after propagation.
The single-plant experimental units were organized in a RCBD with 3 replications. Visual damage scores were taken at three and seven days after inoculation. The controls consisted of untreated susceptible checks (CIAT 36061 and RZ05 3394) inoculated with a sterile agar microdiscs solution $(0.75 \mathrm{~g} / \mathrm{ml}$ PDB). The inoculated susceptible checks were observed daily. Assessments were performed 3, 5, and 7 DAI. The entire experiment was evaluated using the 11-point visual damage scale described previously. Visual damage scores were used to calculate the AUDPC. Differences among genotypes were established based on mean AUDPC by ANOVA, and genotype means were compared by Fisher's protected LSD $(P<0.05)$.

\section{Results}

Comparison of inoculation methods. Temperature and relative humidity inside microchambers were maintained at $24.2 \pm 0.1^{\circ} \mathrm{C}$ and $97.01 \pm 0.2 \%$, respectively. All three inoculum types were successful in causing disease symptoms in both susceptible and resistant plants. In contrast, the absolute control (noninoculated plants) showed no disease symptoms. Resistant and susceptible genotypes
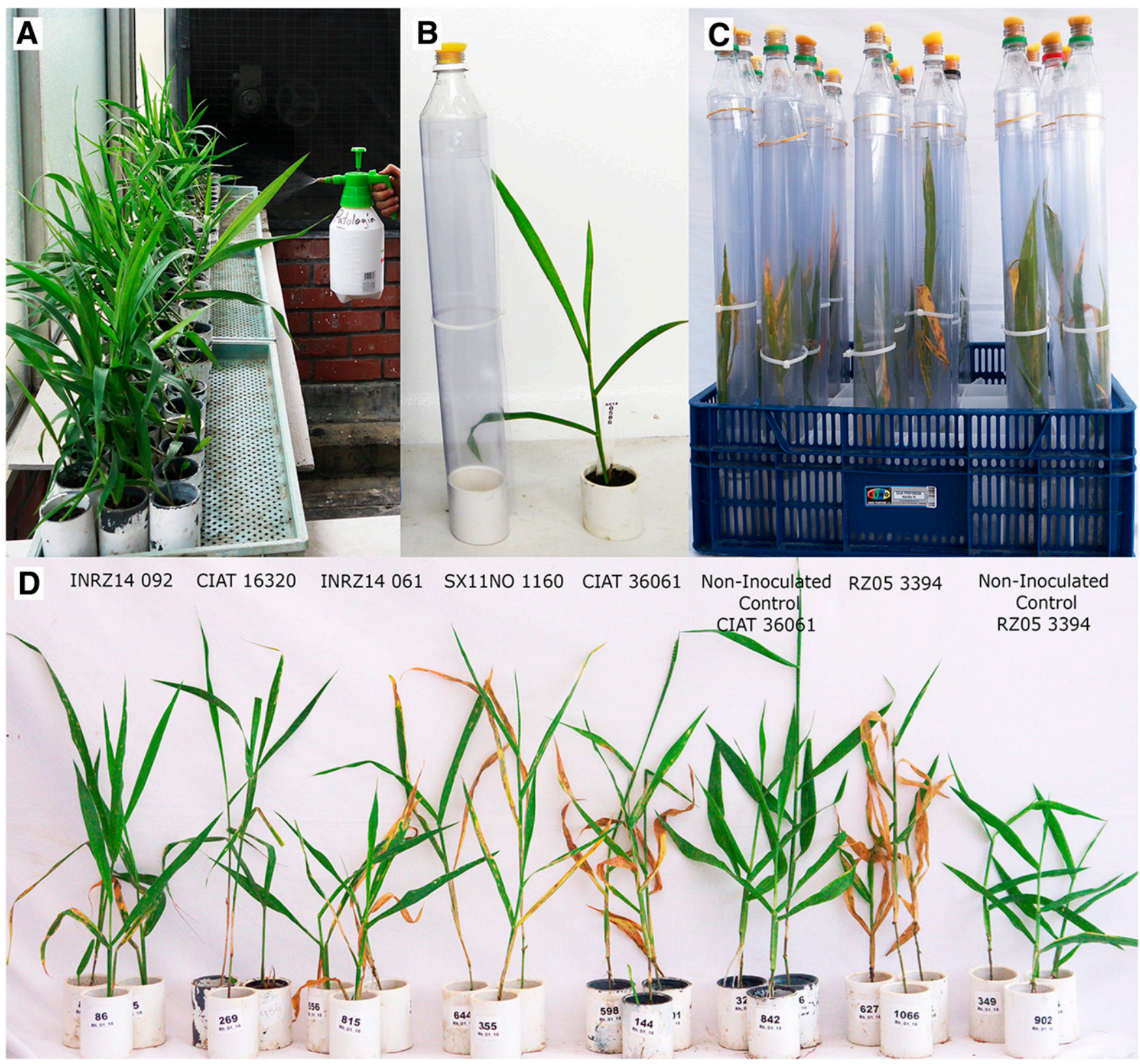

CIAT 3605

RZ05 339

Fig. 1. A, Inoculum being sprayed on Brachiaria plants; B and C, microchambers; and D, resistant and susceptible check genotypes and noninoculated control plants from the validation trial. 
(CIAT 16320 and CIAT 36061) differed for VDS, AUDPC, and ChlL (Table 1, Fig. 2) regardless of inoculum type. Area under the disease progress curve differed for the three inoculum types and among concentrations within inoculum types (Table 1, Fig. 2). In contrast, ChlL differed only for genotype and inoculum type but not among concentrations within inoculum types (Table 1, Fig. 2). For the three VDS evaluation dates (three, five, or seven days after inoculation), ANOVA revealed significant treatment effects as follows: three and five days after inoculation for the main effects of genotype, inoculum type, concentration, and genotype-inoculum type interaction; seven days after inoculation for all sources of variation (Table 1). Percentage chlorophyll loss was moderately correlated with AUDPC $(\mathrm{r}=0.45, P<0.001)$ and VDS seven days after inoculation $(\mathrm{r}=0.47, P<0.001)$.

The resistant genotype, CIAT 16320, presented a higher AUDPC when inoculated with mycelium-infected agar disc(s) $(9.47 \pm 0.7$ for one disc and $8.23 \pm 0.9$ for two discs) and microencapsulated at $1 \mathrm{~g} / \mathrm{ml}$ PDB $(10.68 \pm 0.63)$, compared with the other inoculum types and concentrations (Fig. 2). The susceptible genotype, CIAT 36061, had the highest mean AUDPC (16.70 \pm 0.5$)$ when inoculated with a microencapsulated-mycelium suspension (Inoculum III) at $0.75 \mathrm{~g} / \mathrm{ml}$ PDB. A low AUDPC was observed in susceptible plants (CIAT 36061) inoculated with microdiscs suspensions (Inoculum II; $5.65 \pm 0.6$ at $0.25 \mathrm{~g} / \mathrm{ml} \mathrm{PDB}, 7.35 \pm 0.7$ at $0.5 \mathrm{~g} / \mathrm{ml}$ PDB, $6.6 \pm 0.8$ at $0.75 \mathrm{~g} / \mathrm{ml}$ $\mathrm{PDB}$, and $10.33 \pm 0.6$ at $1 \mathrm{~g} / \mathrm{ml} \mathrm{PDB})$. From ANOVA results, absolute or relative differences between $\mathrm{S}$ and $\mathrm{R}$ controls were observed when plants were inoculated with mycelium-infected agar discs (Inoculum I) or microdiscs suspensions (Inoculum II; Fig. 2). In contrast, resistant and susceptible genotypes were well discriminated when plants were inoculated with microencapsulated-mycelia (Inoculum III), particularly at 0.5 and $0.75 \mathrm{~g} / \mathrm{ml}$ PDB (Fig. 2); at $1 \mathrm{~g} / \mathrm{ml}$ PDB the discrimination is very poor. Additional analyses and ANOVA with both experiments, and the treatments considered in both experiments were performed. The effect of experiment per genotype was tested in order to assess the repeatability of the method in discriminating $S$ and $R$ controls (Table 2, Fig. 3). All the variables evaluated did not show differences between experiments [VDS 3 DAI $(F=0.9, P=0.34)$; VDS 5 DAI $(F=0.01, P=0.93)$; VDS 7 DAI $(F=0.06, P=0.81)$; AUDPC $(F=0.14, P=0.71)$; ChlL $(F=4.76, P=0.04)]$.

Validation of microencapsulated-mycelium suspension method. Three and seven days after inoculation, the entire validation experiment was evaluated. The susceptible checks showed a mean VDS of approximately 4.0 seven days after inoculation. The absolute controls (uninoculated, susceptible checks) exhibited no RFB damage. Area under disease progress curve was normally distributed for the 350 tested genotypes from the SX14 population (Fig. 4). Analysis of variance (AUDPC variable) revealed significant differences among genotypes $(P<0.001)$. Sclerotia formed on infected leaves of some of the most susceptible genotypes. The reaction of check genotypes was consistent with previous observations: the resistant checks (CIAT 16320 and INRZ14/092) had mean AUDPC scores of 5.8 and 6.6, respectively; the intermediate check (INRZ14/061) had a mean AUDPC of 12.4; and the susceptible checks (CIAT 36061 and RZ05/3394) had mean AUDPC of 16.8 and 18.5, respectively.

\section{Discussion}

Results of this study indicate that inoculum type had a marked effect on the severity of RFB on artificially inoculated, greenhousegrown Brachiaria grass plants. Plants can be inoculated by uniformly applying viable spores or fungal mycelium (Russell 2013). Similar results were obtained by Alvarez et al. $(2013,2014)$, when inoculating the genotypes CIAT 36061 and CIAT 16320 with discs and using the same isolate (A-36061) obtained in the present study, discriminating susceptible and resistant genotypes.

Microencapsulated-mycelium solutions (Inoculum III) at 0.75 and $1 \mathrm{~g} / \mathrm{ml}$ PDB produced greater visual damage in the susceptible check

Table 1. Analysis of variance of five response variables assessed for different Rhizoctonia inoculum types tested on resistant and susceptible plants (Trial 1)

\begin{tabular}{|c|c|c|c|c|c|}
\hline Source of variation ${ }^{a}$ & DF & Sum of squares & Mean squares & $F$ value & $\operatorname{Pr}>F^{\mathrm{b}}$ \\
\hline \multicolumn{6}{|l|}{ Visual damage score (3 DAI) } \\
\hline Genotype & 1 & 16.75 & 16.75 & 76.15 & $<0.0001 * *$ \\
\hline IT & 2 & 27.78 & 13.89 & 63.13 & $<0.0001 * *$ \\
\hline Concentration (IT) & 7 & 21.44 & 3.06 & 13.92 & $<0.0001 * *$ \\
\hline Genotype $\times$ IT & 2 & 2.46 & 1.23 & 5.6 & $0.0044 * *$ \\
\hline Genotype $\times$ Concentration (IT) & 7 & 2.10 & 0.30 & 1.36 & $0.2234 \mathrm{~ns}$ \\
\hline \multicolumn{6}{|l|}{ Visual damage score (5 DAI) } \\
\hline Genotype & 1 & 31.10 & 31.10 & 111.25 & $<0.0001 * *$ \\
\hline IT & 2 & 31.00 & 15.50 & 55.44 & $<0.0001 * *$ \\
\hline Concentration (IT) & 7 & 20.14 & 2.88 & 10.29 & $<0.0001 * *$ \\
\hline Genotype $\times$ IT & 2 & 4.07 & 2.04 & 7.28 & $0.0009 * *$ \\
\hline Genotype $\times$ Concentration (IT) & 7 & 4.39 & 0.63 & 2.24 & $0.0331 \mathrm{~ns}$ \\
\hline \multicolumn{6}{|l|}{ Visual damage score (7 DAI) } \\
\hline Genotype & 1 & 46.07 & 46.07 & 156.08 & $<0.0001 * *$ \\
\hline IT & 2 & 40.04 & 20.02 & 67.84 & $<0.0001 * *$ \\
\hline Concentration (IT) & 7 & 17.48 & 2.50 & 8.46 & $<0.0001 * *$ \\
\hline Genotype $\times$ IT & 2 & 6.50 & 3.25 & 11.02 & $<0.0001 * *$ \\
\hline Genotype $\times$ Concentration (IT) & 7 & 7.15 & 1.02 & 3.46 & $0.0017 * *$ \\
\hline \multicolumn{6}{|l|}{ Chlorophyll loss (\%) } \\
\hline Genotype & 1 & 4227.11 & 4227.11 & 25.42 & $<0.0001 * *$ \\
\hline IT & 2 & 1606.22 & 803.11 & 4.83 & $0.0091 * *$ \\
\hline Concentration (IT) & 7 & 2081.57 & 297.37 & 1.79 & $0.0924 \mathrm{~ns}$ \\
\hline Genotype $\times$ IT & 2 & 307.49 & 153.75 & 0.92 & $0.3986 \mathrm{~ns}$ \\
\hline Genotype $\times$ Concentration (IT) & 7 & 1063.83 & 151.98 & 0.91 & $0.4969 \mathrm{~ns}$ \\
\hline \multicolumn{6}{|l|}{ Area under disease progress curve } \\
\hline Genotype & 1 & 793.72 & 793.72 & 159.38 & $<0.0001 * *$ \\
\hline IT & 2 & 890.19 & 445.09 & 89.38 & $<0.0001 * *$ \\
\hline Concentration (IT) & 7 & 585.00 & 83.57 & 16.78 & $<0.0001^{* *}$ \\
\hline Genotype $\times$ IT & 2 & 104.59 & 52.29 & 10.50 & $<0.0001 * *$ \\
\hline Genotype $\times$ Concentration $($ IT) & 7 & 101.71 & 14.53 & 2.92 & $0.0066^{* *}$ \\
\hline
\end{tabular}


than the other inoculum types and allowed for better discrimination among genotypes. Plants inoculated with microdisc suspensions (Inoculum II) showed less damage on average than those inoculated with microencapsulated-mycelium solutions. A possible explanation for this finding is that each particle of microencapsulated-mycelium has potential to cause disease symptoms because the microencapsulated mycelium is prepared by mixing mycelium and agar homogeneously. In contrast, the pathogen is inoculated on agar Petri dishes to generate microdiscs, where the mycelium grows only on the agar surface and does not invade the full depth of the agar. Therefore, when microdiscs suspensions are produced, some of the microdiscs do not contain mycelium. When microencapsulated-mycelium solutions are sprayed on the plants, all above-ground parts of all plants are exposed to the same inoculum density. Agar microparticles allow the pathogen to adhere to the surface of the plant, provide nutrients through the mycelial network to the point of infection, and help prevent drip, drying, or accumulation of inoculum in sheath leaf.

When plants were inoculated with mycelium-infected agar discs (Inoculum I) and microencapsulated-mycelium (Inoculum III) at $1 \mathrm{~g} / \mathrm{ml}$ PDB, higher AUDPC was observed in the resistant checks compared with the other inoculum types. Mycelium-infected agar discs cause the leaf in contact with the disc to rapidly become necrotic and desiccated, even in resistant genotypes. Additionally, the fungal colonization of the host plant is slow in large plants, thus more
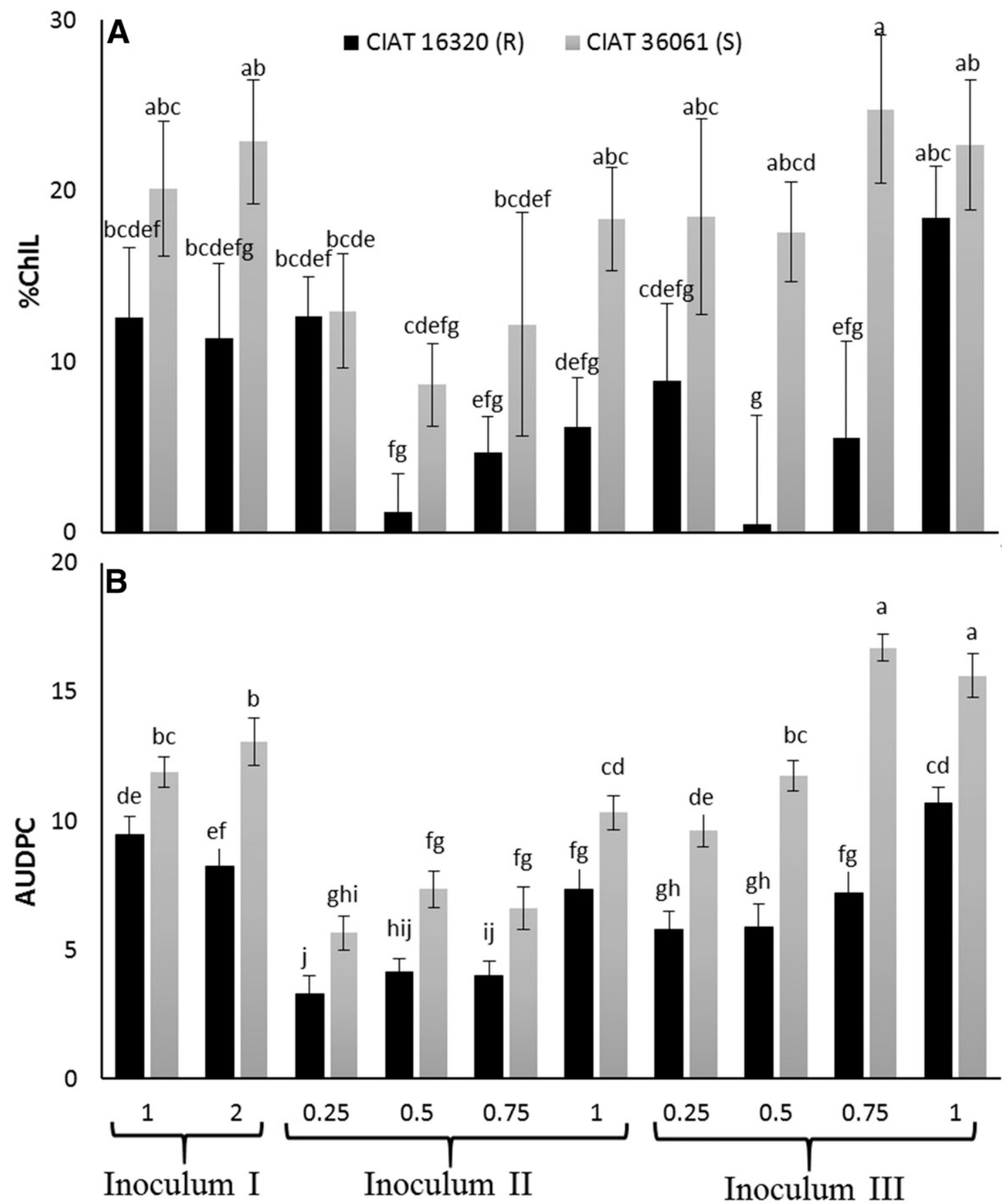

Fig. 2. A, Area under the disease progress curve and B, percentage of chlorophyll loss for different Rhizoctonia inoculum types and concentrations tested on resistant (R, CIAT 16320) and susceptible (S, CIAT 36061) genotypes. Inoculum treatments included mycelium-infected agar disc(s) (Inoculum I), microdisc suspensions (Inoculum II), and microencapsulated-mycelium suspensions (Inoculum III). Inoculum I was tested at dosages of 1 and 2 agar discs per plant, while Inoculum II and III were evaluated at concentrations of $0.25,0.50,0.75$ or $1 \mathrm{~g} / \mathrm{ml}$ PDB. Means not followed by the same letter differ by LSD 0.05 . Least significance differences $\left(\mathrm{LSD}_{0.05}\right)$ values for area under disease progress curve $(\mathrm{AUDPC})=2.01$ and percentage chlorophyll loss $(\mathrm{ChIL})=12.28$. Error bars represent the standard error. 
distal (upper) leaves may escape infection. The microencapsulatedmycelium solution at $1 \mathrm{~g} / \mathrm{ml}$ PDB had a high pathogen concentration, which overwhelmed even moderately resistant genotypes. These factors explain the poor discrimination among genotypes and a reduced ability to distinguish between resistant and susceptible genotypes in these treatments (Fig. 2).

The main aim of artificially inoculating breeding populations is to discriminate among genotypes that have greater or less genetic resistance to the pathogen (Russell 2013). Therefore, once $R$. solani infection has been established, a precise evaluation method is essential for reliable quantification of disease symptoms (Park et al. 2008). Resistance to RFB among genotypes in breeding populations is often quantitative and normally distributed, as observed in the 350 tested genotypes of the SX14 recurrent selection population. The VDS scale used in this study allowed quantification of disease severity, which reflects the level of plant resistance. The positive and significant correlation between AUDPC and ChlL suggests that AUDPC reflects real losses that occur in the plant when attacked by the pathogen and is an appropriate measurement for discrimination between resistant and susceptible genotypes.

Pathogen concentration must be quantified in order to identify and standardize the optimum inoculum dose. A low pathogen concentration can prolong the development of disease symptoms. On the other hand, a high pathogen concentration can overwhelm even moderately resistant genotypes, causing poor discrimination among genotypes. Quantification of pathogen concentration, particularly for a pathogen without spores, such as $R$. solani, is difficult for mycelium-infected agar disc and microdisc solutions. However, for microencapsulated-mycelium, quantification of pathogen concentration is possible and relatively simple. In a previous trial (unpublished data), we measured pathogen concentration and found a highly significant Pearson correlation between mycelium wet weight and dry weight $(\mathrm{r}=0.95 ; P<0.01)$. The conversion equation obtained $(Y=0.1192 X$, where $Y=$ mycelium dry weight and $X=$ mycelium wet weight; $\mathrm{R}^{2}=0.90$ ) allows estimation of the dry weight of microencapsulated mycelium. Therefore, in PDAmicroencapsulated mycelium diluted in PDB $(0.75 \mathrm{~g} / \mathrm{ml} \mathrm{PDB})$, the real dry weight content of mycelium was $9.86 \times 10^{-3} \mathrm{~g} / \mathrm{ml}$ solution, and consequently each plant was inoculated with approximately $0.025 \mathrm{~g}$ mycelium (wet weight) or $0.0029 \mathrm{~g}$ mycelium (dry weight). This concentration was found to be high enough to cause adequate development disease symptoms and low enough to differentiate among genotypes with different degrees of RFB resistance.
Appropriate environmental conditions are necessary to stimulate pathogen growth and penetration (Porta-Puglia \& Aragona 1997). Researchers working in rice pathology have developed strategies to promote environmental conditions suitable for disease development including the microchamber method (with clear plastic bottles) (Jia et al. 2007) and covering the inoculum with aluminum foil (Park et al. 2008). These two methodologies promote high relative humidity, a factor essential for rapid and uniform pathogen growth, which reduces environmental variation. The results of this study suggested that the microchambers promoted environmental conditions necessary for RFB development in Brachiaria spp. And, very importantly, the individual plant microchambers prevent the pathogen's movement between plants, which can create additional, nongenetic variability (J. W. Miles, personal observation).

The aim of the validation method trial was to corroborate the efficiency of the microencapsulated mycelium solution inoculum at a concentration of $0.75 \mathrm{~g} / \mathrm{ml} \mathrm{PDB}$ on large breeding populations. We found that spray-applied microencapsulated-mycelium at $0.75 \mathrm{~g} / \mathrm{ml}$ PDB allowed for differentiation between susceptible and resistant genotypes in a population of 350 tested genotypes from the SX14 recurrent selection population. Potential sources of resistance were identified in the experiment evaluating 350 Brachiaria genotypes (SX14/0153, SX14/0593, SX14/1032, SX14/0907, and SX14/0645 showed an AUDPC average of 8, 8.8, 8.8, 9.4, and 9.5, respectively). In addition, potential susceptible controls were also identified including: SX14/0020, SX14/0366, SX14/0929, SX14/0365, and SX14/ 0927 (showing an average AUDPC of 18.5, 18.5, 18.5, 19 and 18.7, respectively).

The SX14 genotypes with the highest level of resistance could be used as parents in the next cycle of recurrent selection in order to further to increase RFB resistance in the Brachiaria spp. breeding program. Comparison with field resistance is needed to assess the capacity of the method to predict field resistance. The value of a phenotyping method resides first in its capacity to predict the level of field resistance. Further research should be conducted to validate that the results from greenhouse trials with microencapsulated mycelium solution at a concentration of $0.75 \mathrm{~g} / \mathrm{ml}$ PDB are well correlated with resistance in replicated field trials, as genotype-environment interaction may modify the response of Brachiaria genotypes to $R$. solani.

In summary, the best results for discrimination of variation in RFB reaction among Brachiaria genotypes under greenhouse conditions were obtained with microencapsulated-mycelium at $0.75 \mathrm{~g} / \mathrm{ml}$

Table 2. Analysis of variance of five response variables assessed for different Rhizoctonia inoculum types tested on resistant and susceptible plants in the confirmation trial for experiment 1

\begin{tabular}{|c|c|c|c|c|c|}
\hline Source of variation ${ }^{a}$ & DF & Sum of Squares & Mean squares & $F$ value & $\operatorname{Pr}>F^{\mathbf{b}}$ \\
\hline \multicolumn{6}{|c|}{ Visual Damage score (3DAI) } \\
\hline Genotype & 1 & 4.08 & 4.08 & 42.22 & $<0.0001 * *$ \\
\hline IT & 2 & 0.32 & 0.16 & 1.67 & $0.201 \mathrm{~ns}$ \\
\hline Genotype x IT & 2 & 0.95 & 0.47 & 4.90 & $0.012 \mathrm{~ns}$ \\
\hline \multicolumn{6}{|c|}{ Visual damage score (5DAI) } \\
\hline Genotype & 1 & 13.55 & 13.55 & 70.30 & $<0.0001 * *$ \\
\hline IT & 2 & 2.01 & 1.01 & 5.22 & $0.0095 * *$ \\
\hline Genotype x IT & 2 & 2.84 & 1.42 & 7.38 & $0.0018 * *$ \\
\hline \multicolumn{6}{|c|}{ Visual damage score (7DAI) } \\
\hline Genotype & 1 & 24.08 & 24.08 & 174.02 & $<0.0001 * *$ \\
\hline IT & 2 & 3.88 & 1.94 & 14.00 & $<0.0001 * *$ \\
\hline Genotype x IT & 2 & 9.04 & 4.52 & 32.67 & $<0.0001 * *$ \\
\hline \multicolumn{6}{|l|}{ Chlorophyll loss (\%) } \\
\hline Genotype & 1 & $10,204.87$ & $10,204.87$ & 30.59 & $<0.0001 * *$ \\
\hline IT & 2 & 501.33 & 250.66 & 0.75 & $0.4780 \mathrm{~ns}$ \\
\hline Genotype x IT & 2 & 55.96 & 27.98 & 0.08 & $0.4969 \mathrm{~ns}$ \\
\hline \multicolumn{6}{|c|}{ Area under disease progress curve } \\
\hline Genotype & 1 & 406.88 & 406.88 & 100.40 & $<0.0001 * *$ \\
\hline IT & 2 & 51.38 & 25.69 & 6.34 & $0.0039 * *$ \\
\hline Genotype x IT & 2 & 85.66 & 42.83 & 10.57 & $0.0002 * *$ \\
\hline
\end{tabular}

a IT = Inoculum type; Genotype $\times$ IT $=$ interaction of genotype with IT.

${ }^{\mathrm{b} * *}$ Effect differs from zero at the $\alpha=0.01$ probability level; ns, no significant treatment effect. 

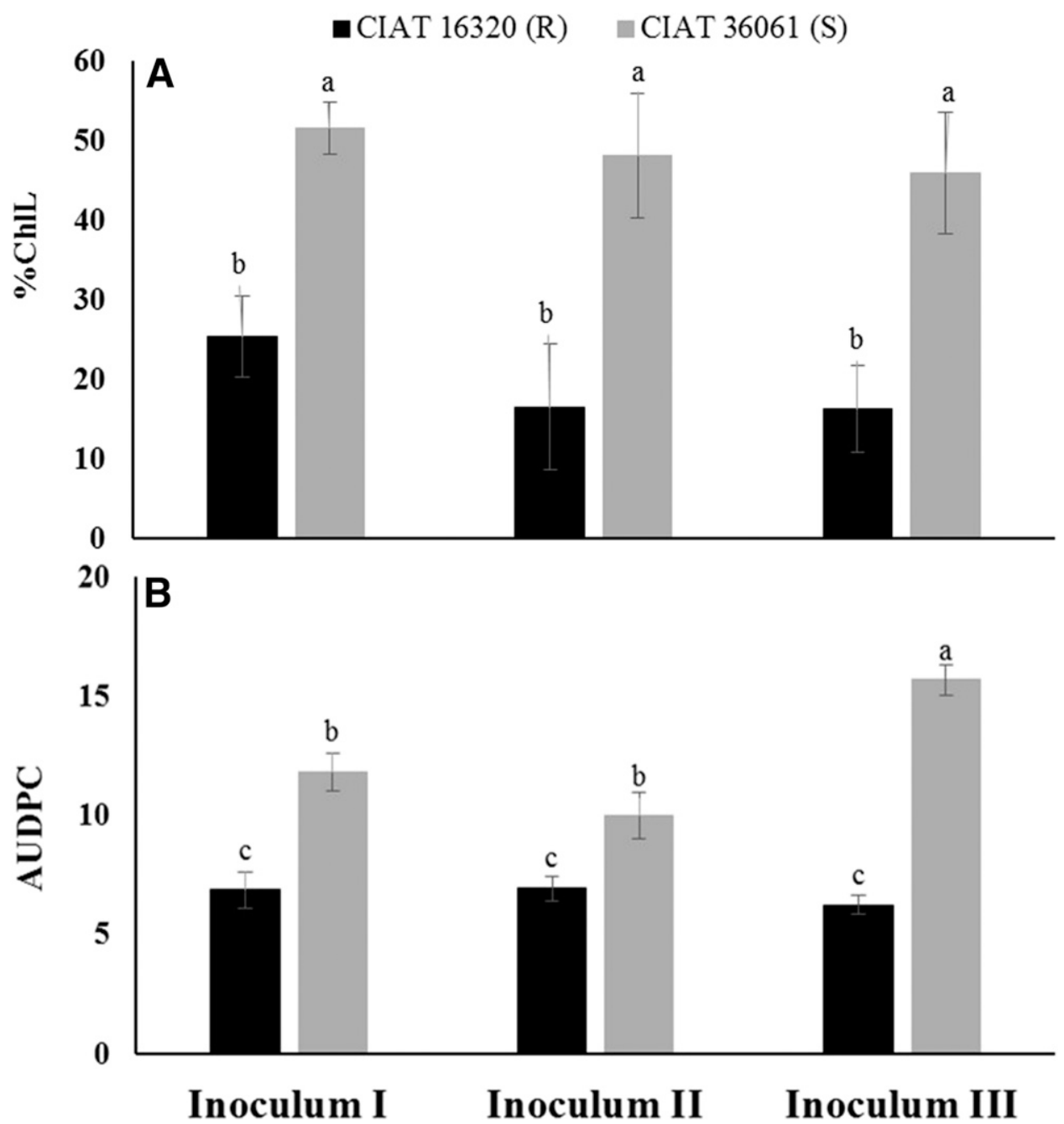

Fig. 3. A, Area under the disease progress curve and $\mathrm{B}$, percentage of chlorophyll loss obtained in the second confirmation experiment for different Rhizoctonia inoculum types tested on resistant (R, CIAT 16320) and susceptible (S, CIAT 36061) genotypes. Inoculum treatments included mycelium-infected agar disc(s) (Inoculum I), microdisc suspensions (Inoculum II), and microencapsulated-mycelium suspensions (Inoculum III). Inoculum I was tested at dosages of two agar discs per plant, while Inoculum II and III were evaluated at concentrations 0.75 or $1 \mathrm{~g} / \mathrm{ml} \mathrm{PDB}$. Means followed by the same letter are not significantly different according to LS mean differences Student's $\mathrm{t}(\alpha=0.05)$. Error bars represent the standard error.

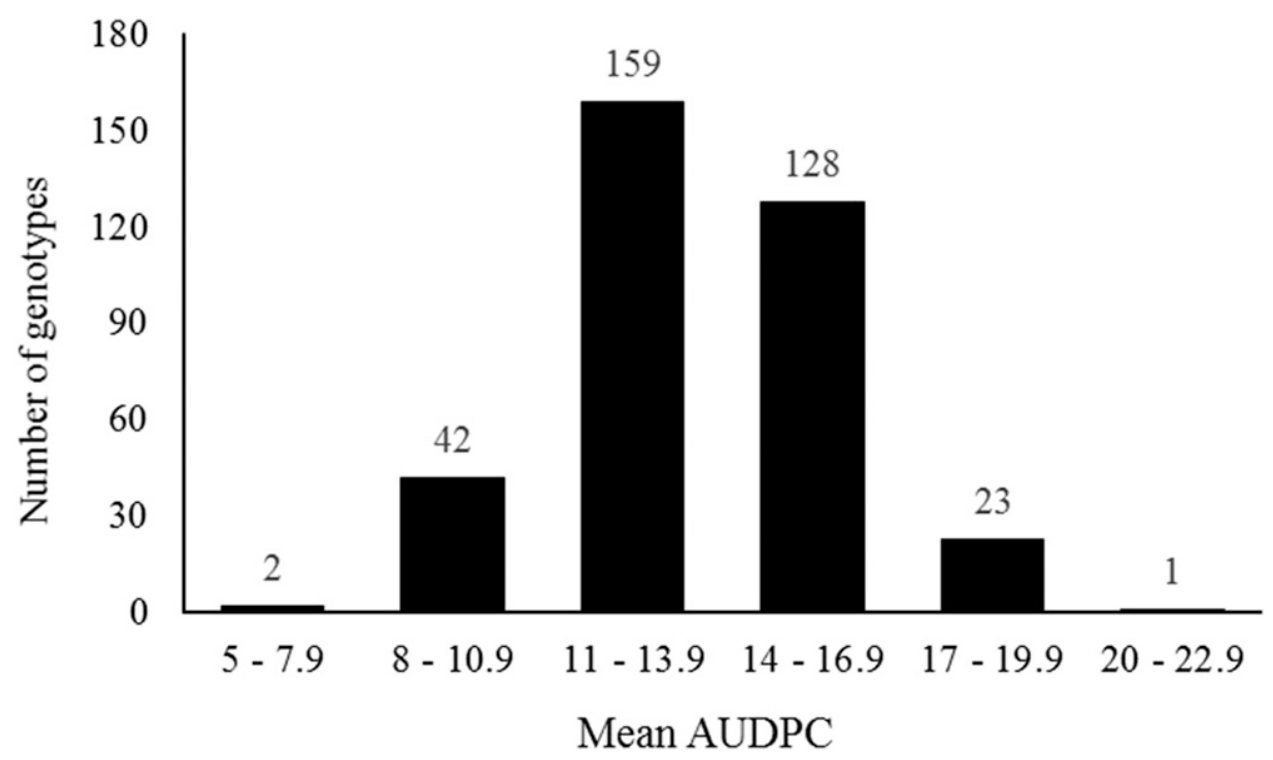

Fig. 4. Distribution of area under disease progress curve (AUDPC) of the 350 SX14 genotypes and five resistant and susceptible check genotypes when inoculated with microencapsulated-mycelium at $0.75 \mathrm{~g} / \mathrm{ml} \mathrm{PDB}$. Mean AUDPC for CIAT $16320=5.8$, INRZ14/092 = 6.6 (resistant checks), INRZ14/061 = 12.4 (intermediate check), CIAT $36061=16.8$, and RZ05/3394 = 18.5 (susceptible checks). 
PDB sprayed on plants. This new protocol has several advantages for RFB evaluation: high throughput capacity in a short time ( 35 days from planting to evaluation); efficient use of space (125 plants $\left./ \mathrm{m}^{2}\right)$; rapid inoculation (approximately 325 plants person per hour); visual evaluation of damage (approximately 95 plants person per hour); ability to quantify the inoculum concentration; and consistent results over replicate trials. This methodology could be implemented in other crops in the grass family that are affected by RFB (e.g., rice) and might even be adapted for other fungal pathogens.

\section{Acknowledgments}

This study was supported by Tropical Forages Program, CGIAR Research Program 3.7 (Livestock and Fish), and Dow AgroSciences. We thank Jeison Velasco and William Mera of Forages Entomology for their assistance in conducting these trials. We thank Paola Sotelo for her critical review of this paper.

\section{Literature Cited}

Alvarez, E., Latorre, M., Bonilla, X., Sotelo, G., and Miles, J. W. 2013. Diversity of Rhizoctonia spp. causing foliar blight on Brachiaria in Colombia and evaluation of Brachiaria genotypes for foliar blight resistance. Plant Dis. 97:772-779.

Alvarez, E., Latorre, M., Bonilla, X., Sotelo, G., and Miles, J. W. 2014. Assessing the resistance of Brachiaria hybrids to pathogenic Rhizoctonia. Plant Dis. 98: 306-310.

Araújo, L. G., Prabhu, A. S., and da Silva, G. B. 2007. Field and greenhouse inoculation methods for assessment of sheath blight resistance in rice. Crop Breed. Appl. Biotechnol. 7:221-224.

Cardona, C., Miles, J. W., and Sotelo, G. 1999. An improved methodology for massive screening of Brachiaria spp. genotypes for resistance to Aeneolamia varia (Homoptera: Cercopidae). J. Econ. Entomol. 92:490-496.

Cardona, C., Miles, J. W., Zuñiga, E., and Sotelo, G. 2010. Independence of resistance in Brachiaria spp. to nymphs or to adult spittlebugs (Hemiptera: Cercopidae): implications for breeding for resistance. J. Econ. Entomol. 103: 1860-1865.

Chongo, G., and Bernier, C. C. 2000. Effects of host, inoculum concentration, wetness duration, growth stage, and temperature on anthracnose of lentil. Plant Dis. 84:544-548.

Jank, L., Barrios, S. C., do Valle, C. B., Simeão, R. M., and Alves, G. F. 2014. The value of improved pastures to Brazilian beef production. Crop Pasture Sci. 65: 1132-1137.

Jia, Y., Correa-Victoria, F., McClung, A., Zhu, L., Liu, G., Wamishe, Y., Xie, J., Marchetti, M. A., Pinson, S. R. M., Rutger, J. N., and Correll, J. C. 2007. Rapid determination of rice cultivar responses to the sheath blight pathogen Rhizoctonia solani using a micro-chamber screening method. Plant Dis. 91:485-489.

Jiménez, J. C., Cardoso, J. A., Arango-Londoño, D., Fischer, G., and Rao, I. 2015. Influence of soil fertility on waterlogging tolerance of two Brachiaria grasses. Agron. Colomb. 33:20-28.

Kelemu, S., Miles, J. W., Bonilla, X. P., and Badel, J. L. 1995. Sources of resistance in species of Brachiaria to foliar blight disease caused by Rhizoctonia solani. Trop. Grassl. 29:257-262.

Loch, D. S., and Miles, J. W. 2002. Brachiaria ruziziensis x Brachiaria brizantha. Brachiaria. 'Mulato'. Plant Var. J. 15:20-21.

Miles, J. W. 2007. Apomixis for cultivar development in tropical forage grasses. Crop Sci. 47:S238-S249.

Miles, J. W., Cardona, C., and Sotelo, G. 2006. Recurrent selection in a synthetic Brachiaria grass population improves resistance to three spittlebug species. Crop Sci. 46:1088-1093.

Miles, J. W., Do Valle, C. B., Rao, I. M., and Euclides, V. P. 2004. Brachiaria grasses. Page 745-783 in: Warm-season (C4) Grasses. L. E. Moser, D. R.
Buxton, and L. E. Sollenberger, eds. American Society of Agronomy, Madison, WI.

Pabón, A., Cardona, C., Miles, J. W., and Sotelo, G. 2007. Response of resistant and susceptible Brachiaria spp. genotypes to simultaneous infestation with multiple species of spittlebugs (Hemiptera: Cercopidae). J. Econ. Entomol. 100:1896-1903.

Park, D. S., Sayler, R. J., Hong, Y.-G., Nam, M. H., and Yang, Y. 2008. A method for inoculation and evaluation of rice sheath blight disease. Plant Dis. 92:25-29.

Peters, M., Franco, L. H., Schmidt, A., and Hincapie, B. 2011. Especies forrajeras multipropósito: Opciones para productores del Trópico Americano. Centro Internacional de Agricultura Tropical (CIAT), Cali, Colombia.

Pineda, J., Hernández, A., González, A., Barrientos, V., Nass, H., and Gil, E. 2005. Técnica de inoculación rápida y eficiente para la evaluación de materiales de maíz ante Rhizoctonia solani kühn. Bioagro. 17:93-98.

Porta-Puglia, A., and Aragona, M. 1997. Improvement of grain legumes general part: Diseases. Field Crops Res. 53:17-30.

Rao, I., Miles, J., Wenzl, P., Louw-Gaume, A., Cardoso, J. A., Ricaurte, J., Polania, J., Rincon, J., Hoyos, V., Frossard, E., Wagatsuma, T., and Horst, W. 2011. Mechanisms of adaptation of Brachiaria grasses to abiotic stress factors in the tropics. Pages 361-383 in:Proceedings of the International Symposium on Forage Breeding, Empresa Brasileira de Pesquisa Agropecuária, Campo Grande, MS, Brazil.

Russell, G. E. 2013. Plant Breeding for Pest and Disease Resistance: Studies in the Agricultural and Food Sciences. Butterworth, Boston.

Shaner, G., and Finney, R. E. 1977. The effect of nitrogen fertilization on the expression of slow-mildewing resistance in Knox wheat. Phytopathology 67: 1051-1056.

Soreng, R. J., Peterson, P. M., Romaschenko, K., Davidse, G., Zuloaga, F. O. Judziewicz, E. J., Filgueras, T. S., Davis, J. I., and Morrone, O. 2015. A worldwide phylogenetic classification of the Poaceae (Gramineae). J. Syst. Evol. 53:117-137.

Sotelo, G., Segura, G., Bonilla, J., and Miles, J. W. 2007. Develop and refine a robust, high throughput screening methodology for reaction to Rhizoctonia solani, based on greenhouse-grown plants and artificial inoculation. Page 16 in: 2007 Annual Report of Project IP-5, Tropical and Grasses Legumes: Optimizing Genetic Diversity for Multipurpose Use. Centro Internacional de Agricultura Tropical (CIAT), Cali, Colombia.

Srinivasachary, S., Beligan, G., Willocquet, L., and Savary, S. 2013. A strategy to identify sources of quantitative resistance in pathosystems involving disease escape and physiological resistance: The case study of rice sheath blight. Plant Pathol. 62:888-899.

Srinivasachary, S., Willocquet, L., and Savary, S. 2011. Resistance to rice sheath blight (Rhizoctonia solani Kühn) [(teleomorph: Thanatephorus cucumeris (A.B. Frank) Donk.] disease: Current status and perspectives. Euphytica 178: $1-22$.

Thakur, R. 2007. Host plant resistance to diseases: Potential and limitations. Indian J. Plant Prot. 35:17-21

Trapero, C., Díez, C. M., Rallo, L., Barranco, D., and López-Escudero, F. J. 2013. Effective inoculation methods to screen for resistance to Verticillium wilt in olive. Sci. Hortic. (Amsterdam) 162:252-259.

Tsror, L. 2010. Biology, epidemiology and management of Rhizoctonia solani on potato. J. Phytopathol. 158:649-658

Valerio, J. R., Lapointe, S. L., Kelemu, S., Fernandez, C. D., and Morales, F. 1996. Pests and diseases of Brachiaria species. Pages 87-105 in: Brachiaria: Biology, Agronomy, and Improvement. J. W. Miles, B. L. Maass, and C. Do Valle, eds. CIAT, EMBRAPA/CNPGC, Cali, Colombia.

Wallwork, H. 2009. The use of host plant resistance in disease control. Pages 122-141 in: Disease Control in Crops: Biological and Environmentally Friendly Approaches. D. Walters, ed. Wiley-Blackwell, Chichester, UK.

Willocquet, L., Lore, J. S., Srinivasachary, S., and Savary, S. 2011. Quantification of the components of resistance to rice sheath blight using a detached tiller test under controlled conditions. Plant Dis. 95:1507-1515. 